\title{
Chronic Treatment with Squid Phosphatidylserine Activates Glucose Uptake and Ameliorates TMT-Induced Cognitive Deficit in Rats via Activation of Cholinergic Systems
}

\author{
Hyun-Jung Park, ${ }^{1}$ Seung Youn Lee, ${ }^{2}$ Hyun Soo Shim, ${ }^{1}$ Jin Su Kim, ${ }^{3}$ \\ Kyung Soo Kim, ${ }^{2}$ and Insop Shim ${ }^{1}$ \\ ${ }^{1}$ Acupuncture and Meridian Science Research Center, Kyung Hee University, Seoul 130-701, Republic of Korea \\ ${ }^{2}$ Department of Family Medicine, The Catholic University of Korea, Seoul 137-701, Republic of Korea \\ ${ }^{3}$ Molecular Imaging Research Center, Korea Institute of Radiological \& Medical Sciences, \\ University of Science \& Technology, Republic of Korea
}

Correspondence should be addressed to Kyung Soo Kim, kskim@catholic.ac.kr and Insop Shim, ishim@catholic.ac.kr

Received 28 November 2011; Revised 6 February 2012; Accepted 6 February 2012

Academic Editor: William C. S. Cho

Copyright (C) 2012 Hyun-Jung Park et al. This is an open access article distributed under the Creative Commons Attribution License, which permits unrestricted use, distribution, and reproduction in any medium, provided the original work is properly cited.

The present study examined the effects of squid phosphatidylserine (Squid-PS) on the learning and memory function and the neural activity in rats with TMT-induced memory deficits. The rats were administered saline or squid derived Squid-PS (SquidPS $50 \mathrm{mg} \mathrm{kg}^{-1}$, p.o.) daily for 21 days. The cognitive improving efficacy of Squid-PS on the amnesic rats, which was induced by TMT, was investigated by assessing the passive avoidance task and by performing choline acetyltransferase (ChAT) and acetylcholinesterase (AchE) immunohistochemistry. 18F-Fluorodeoxyglucose and performed a positron emission tomography (PET) scan was also performed. In the passive avoidance test, the control group which were injected with TMT showed a markedly lower latency time than the non-treated normal group $(P<0.05)$. However, treatment of Squid-PS significantly recovered the impairment of memory compared to the control group $(P<0.05)$. Consistent with the behavioral data, Squid-PS significantly alleviated the loss of ChAT immunoreactive neurons in the hippocampal CA3 compared to that of the control group $(P<0.01)$. Also, SquidPS significantly increased the AchE positive neurons in the hippocampal CA1 and CA3. In the PET analysis, Squid-PS treatment increased the glucose uptake more than twofold in the frontal lobe and the hippocampus $(P<0.05$, resp.). These results suggest that Squid-PS may be useful for improving the cognitive function via regulation of cholinergic enzyme activity and neural activity.

\section{Introduction}

Trimethyltin (TMT) is an organotin compound with potent neurotoxicant effects. This substance is regarded as being particularly useful for studying the response to injury on account of the distinct pattern of degeneration it causes in rodent brain. In particular, the rat hippocampus constitutes the most suitable model for TMT-induced brain injury [14]. The molecular basis for the selective vulnerability of specific neuronal populations to neuronal insults has been a key focus in the fields of neurology and neuropathology [5]. TMT-induced neurodegeneration is characterized by massive neuronal death that is mainly localized in the limbic system and especially in the hippocampus, and this is accompanied by reactive gliosis, epilepsy, and marked neurobehavioral alterations, and so this is considered a useful model of neurodegeneration and selective neuronal death [5-11]. Also, in rats, TMT induces the degeneration of pyramidal neurons in the hippocampus and the cortical areas (pyriform cortex, entorhinal cortex, and subiculum) connected to the hippocampus, but there is also neuronal loss in the association areas $[6,12,13]$. Furthermore, behavioral studies have shown increased locomotor activity, disruption in self-grooming, and learning deficits in TMT-intoxicated rats $[1,5,14-21]$. TMT intoxication impairs the performance of learning acquisition of water maze and Biel maze (water avoidance) tasks as well as the performance of HebbWilliams maze and radial arm maze tasks. In addition, TMT 
intoxication produces deficits in passive avoidance retention, but not in the acquisition of the passive avoidance response $[2-4,10,21,22]$. Furthermore, deficits in the acquisition of active avoidance at the beginning of training have been reported [16]. Moreover, TMT has been shown to produce effects on operant behavior since TMT-intoxicated rats had higher rates of lever pressing under a fixed-ratio schedule of food presentation [20], and TMT impaired the performance of differential reinforcement at low response rates in an operant schedule [23]. These anatomical and behavioral findings have made TMT-intoxicated rats an attractive model for degenerative diseases such as $\mathrm{AD}$, which is the most common cause of dementia [16].

Phosphatidylserine (PS), a phospholipid nutrient, is active in cell membranes and is the major acidic phospholipid component in the membranes of the brain. Membranes are the working surfaces of every cell, carrying out the essential functions of cellular communication and hormonal signal transduction [24, 25]. Nerve cells, in particular, depend on healthy membrane function for normal neurotransmitter metabolism and nerve signal transmission [26]. Also, PS assists in maintaining adequate glucose utilization in the brain. Glucose is the preferred energy substrate for nerve cells which, unlike other cells, are unable to use fatty acids or proteins for energy production. Brain glucose utilization, an indicator of brain activity, often declines during aging [27].

The present study was undertaken to evaluate the neuroprotective effect of Squid-PS on the TMT-induced memory deficit in rats and to elucidate the mechanism underlying these protective effects in rats. Rats were tested on a passive avoidance test for learning and memory. The analyzed parameters included the expression of cholinergic neurons and neural activity in the hippocampus.

\section{Materials and Methods}

2.1. Animals and the Experimental Design. Male SpragueDawley rats weighting 250-280 g (8 weeks old) each were purchased from Samtaco Animal Corp. (Kyungki-do, Korea). The animals were housed in individual cages under lightcontrolled conditions (12/12-hour light/dark cycle) and at $23^{\circ} \mathrm{C}$ room temperature. Food and water were made available ad libitum. All the experiments were approved by the Kyung Hee University institutional animal care and use committee. Also, this experimental protocol was approved by an Institutional Review Committee for the Use of Human or Animal Subjects or that procedures are in compliance with at least the Declaration of Helsinki for human subjects, the National Institutes of Health Guide for Care and Use of Laboratory Animals (Publication no. 85-23, revised 1985), the UK Animals Scientific Procedures Act 1986, or the European Communities Council Directive of 24 November 1986 (86/609/EEC). The rats were allowed at least 1 week to adapt to their environment before the experiments.

The rats were injected intraperitoneally (i.p.) with TMT $(8.0 \mathrm{mg} / \mathrm{kg}$, body weight) dissolved in $0.9 \%$ saline, and then they were returned to their home cages.

The rats were randomly assigned to three groups of six individuals each as follows: nontreated, naïve normal group (normal); saline-treated group (control); $50 \mathrm{mg} \mathrm{kg}^{-1}$ SquidPS-treated group (Squid-PS 50) used in this study, which were manufactured and kindly provided by Doosan Co. Glonet BU (Youngin, Korea). The rats were orally administrated with PS, daily for 21 days.

2.2. Passive Avoidance Task (PAT). A passive avoidance task was performed after 21 days of the administration of SquidPS. Rats were trained in a step-through inhibitory avoidance task. On the training trial, each rat was placed on a lighted platform outside a hole leading to a dark compartment. When the rat stepped into the dark compartment, a constant current foot shock ( $5 \mathrm{~V}, 0.5 \mathrm{~mA}, 10$ seconds) was delivered twice. For the retention test, at 24 hours (day1), and at 2 and 3 days later, each rat was again placed on the platform, and the latency to step through was recorded.

2.3. Immunohistochemistry. Briefly, the rats were anesthetized (sodium pentobarbital, $100 \mathrm{mg} / \mathrm{kg}$, IP) and then perfused transcardially with heparinized phosphate-buffered saline (PBS; $\mathrm{pH} 7.4$ ) for $30 \mathrm{~min}$ followed by $4 \%$ paraformaldehyde in $0.1 \mathrm{M}$ phosphate buffer ( $\mathrm{pH}$ 7.4) for 10-15 min. The brains were postfixed in the same fixative overnight, cryoprotected in 30\% sucrose solution in PBS, embedded and serially sectioned on a cryostat (Leica, Germany) at $30 \mu \mathrm{m}$ thickness in the coronal plane, and were collected in PBS. The primary antibodies against the following specific antigen were used: cholinacetyl transferase (sheep polyclonal ChAT, concentration 1:2000; Cambridge Research Biochemicals, Cleveland, UK) and acetylcholinesterase (rabbit polyclonal AchE, concentration 1: 1000; Cambridge Research Biochemicals, Cleveland, UK). The primary antibody was prepared and diluted in $0.3 \%$ PBST, $2 \%$ blocking serum, and $0.001 \%$ kehole limpet hemocyanin (Sigma, USA). The sections were incubated in the primary antiserum for $72 \mathrm{~h}$ at $4^{\circ} \mathrm{C}$. Following rinsing in PBST, sections were incubated for $2 \mathrm{hr}$ at room temperature in biotinylated rabbit anti-sheep or antirabbit serum (Vector Laboratories, Burlingame, CA, USA) diluted 200X in PBST containing $2 \%$ normal rabbit serum. Following a further rinsing in PBS, the tissue was developed using diaminobenzidine (Sigma, USA) as the chromogen. The images were captured using a DP2-BSW imaging system (Olympus, CA, USA), and they were processed using Adobe Photoshop. For measuring the cells that were positive for ChAT and AchE, the grid was placed on CA1 and CA 3 in the hippocampus area according to the method of Paxinos et al. [28]. The number of cells was counted at 100x magnification using a microscope rectangle grid that measured $200 \times$ $200 \mathrm{~mm}$. The cells were counted in three sections per rat within the hippocampal CA1 and CA 3 areas. The brain sections were visually inspected at 3 different anteroposterior levels extending from -2.12 to $-6.04 \mathrm{~mm}[28]$.

2.4. [F-18]FDG Micro-PET Scan. All the rats were deprived of food for $12-15 \mathrm{~h}$ before the experiments to enhance the [F18] FDG uptake in the brain [20]. Each animal was placed on a heating pad in a cage and warmed for at least $30 \mathrm{~min}$ before the [F-18] FDG injection. The temperature of the cages was kept at $30^{\circ} \mathrm{C}$ throughout the uptake period in accordance 
with an optimized [F-18] FDG uptake protocol [20]. [F-18] FDG $(500 \mu \mathrm{Ci} / 100 \mathrm{~g}$ body weight $)$ was injected through a tail vein, and the rats were anesthetized with $2 \%$ isoflurane in 100\% oxygen (Forane solution; ChoongWae Pharma). For the PET imaging, a Siemens Inveon PET scanner (Siemens Medical Solutions, USA) was used throughout the study. The transverse resolution that was used was $<1.8 \mathrm{~mm}$ at the center [20, 29].

The transmission PET data was acquired for $15 \mathrm{~min}$ using a Co-57 point source with an energy window of $120 \sim 125 \mathrm{keV}$. One mCi of [F-18] FDG was injected. After allowing for $30 \mathrm{~min}$ of tracer uptake time, $30 \mathrm{~min}$ of emission PET data was acquired within an energy window of 350 $650 \mathrm{keV}$. The emission list-mode PET data was sorted into $3 \mathrm{D}$ sinograms and reconstructed using 3 DRP methods. The pixel size of the reconstructed image was $0.15 \times 0.15 \times$ $0.79 \mathrm{~mm}^{3}$. Attenuation and scatter corrections were performed for all the datasets. Automated regions of interest (ROI) were used to sample cerebral metabolic rate for glucose from the spatially normalized PET within specific ADrelated brain regions (hippocampus, prefrontal cortex).

2.5. Voxel-Based Statistical Analysis. Voxel-based statistical analysis was performed to compare the cerebral glucose metabolism of the Squid-PS and control datasets. The procedure used for SPM analysis of the animal PET data was as previously described in our previous study [29]. Briefly, for efficient spatial normalization, only the brain region was extracted. A study-specific template was then constructed using all the datasets. The PET data was spatially normalized onto a rat brain template and smoothed using a $3 \mathrm{~mm}$ Gaussian kernel. Count normalization was performed. A voxelwised $t$-test between the Squid-PS and normal datasets was performed using the Statistical Parametric Mapping 5 pro$\operatorname{gram}(P<0.05, K>50)$.

2.6. Statistical Analysis. Statistical comparisons were done for the behavioral and histochemical studies using one-way or two-way ANOVA and repeated measures of ANOVA, respectively, and Tukey's post hoc test was done. All of the results are presented as means \pm SEM, and SPSS 15.0 for Windows was used for analysis of the statistics. The significance level was set at $P<0.05$.

\section{Results}

3.1. Effect of Squid-PS on the Performance of the Passive Avoidance Task. As shown in Figure 1, the control group showed a memory deficit in the passive avoidance test compared to the normal group $\left(F_{2,11}=2.68, P<0.05\right)$. However, the Squid-PS group showed improved memory compared to the control (Day 3, $P<0.01$ ).

3.2. ChAT Immunoreactive Neurons of the Hippocampus. ChAT immunoreactive cells in the different hippocampal subregions are shown in Figure 2. Post-hoc comparisons indicated that the ChAT immunoreactivity in the hippocampal CA1 and CA3 of the control group was significantly reduced compared with that of the normal group $\left(F_{2,16}=5.4\right.$,

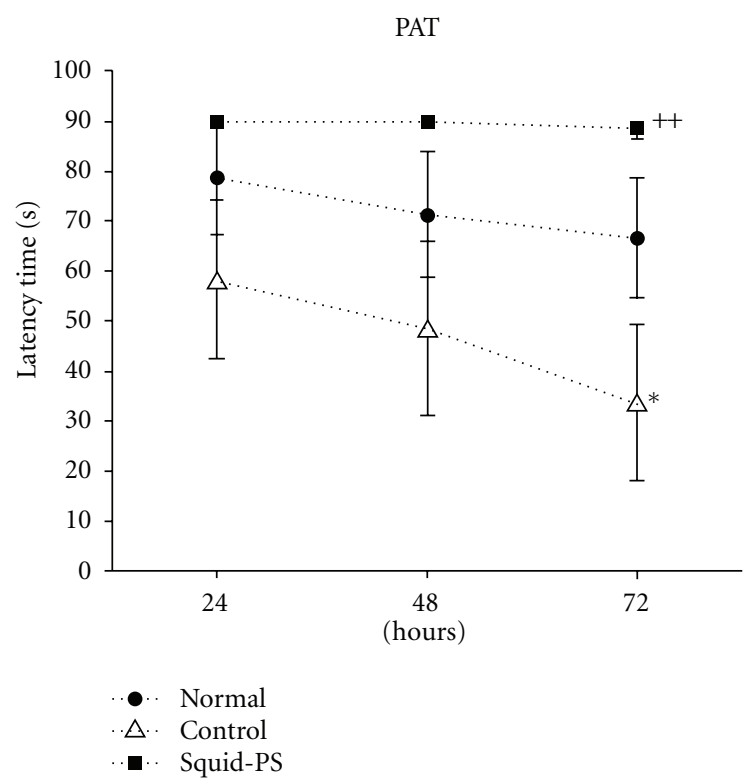

FIgure 1: The effect of Squid-PS on passive avoidance task. Data represent means \pm SEM of the latency in the passive avoidance task, on learning and memory performance. Rats were exposed to the acquisition trial (DAY1), and retention tests were performed on the following two days (DAY2-3). Statistics: one-way ANOVA test, followed by LSD test. ${ }^{*} P<0.05$ as compared with the corresponding data of normal group. ${ }^{++} P<0.01$ as compared with the corresponding data of control group.

$\left.P<0.05 ; F_{2,16}=15.6, P<0.001\right)$. Also, ChAT reactivity in the Squid-PS group was higher than that of the control group, and particularly in CA3 $(P<0.01)$.

3.3. AchE Immunoreactive Neurons of the Hippocampus. AchE immunoreactive cells in the different hippocampal subregions are shown in Figure 3. Post hoc comparisons indicated that the AchE activity in the hippocampus of the control group was significantly lower than that of the normal group $(P<0.01)$. In particular, there were significant differences in both CA1 $\left(F_{2,13}=7.3, P<0.01\right)$ and CA3 $\left(F_{2,13}=\right.$ $7.6, P<0.01)$. However, the AchE reactivity in the SquidPS group was higher than that of the control group, and particularly in CA1 $(P<0.01)$ and CA3 $(P<0.05)$.

3.4. Change in Brain Glucose Metabolism. The analysis of the brain glucose metabolism analysis was shown in Figure 4, and Table 1. The result of voxel-wised shows comparison between the Squid-PS and control datasets. FDG-PET image scans indicated differences in the cerebral metabolic rate of glucose from the hippocampus to prefrontal cortices between the control and Squid-PS group. On the SPM analysis, the glucose metabolism of the Squid-PS datasets was significantly increased in the hippocampus and frontal lobe compared to that of the control $(P<0.05)$.

\section{Discussion}

The present study demonstrated that TMT injections produced severe deficits in the rats' performances in a passive 


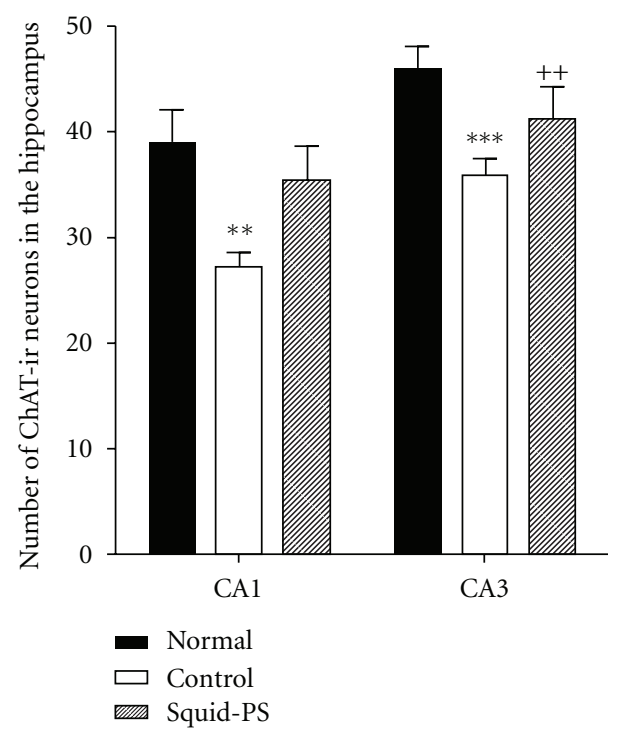

(a)

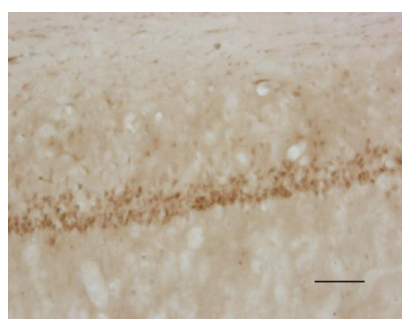

(b)

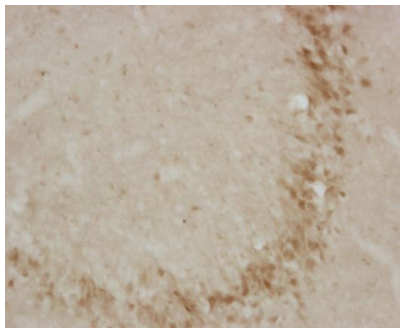

(e)

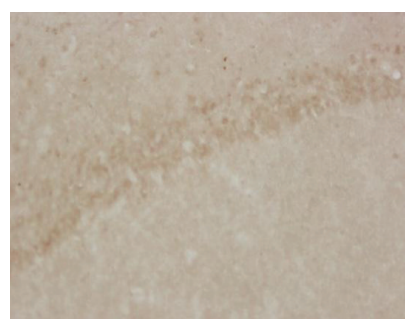

(c)

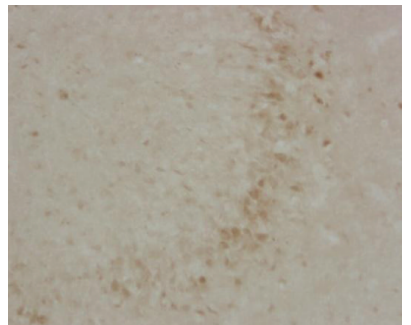

(f)

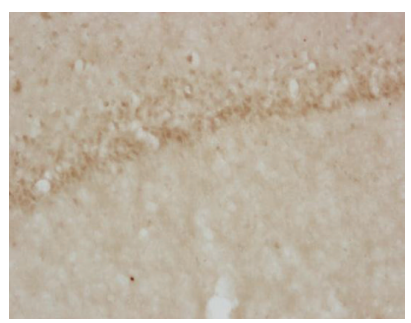

(d)

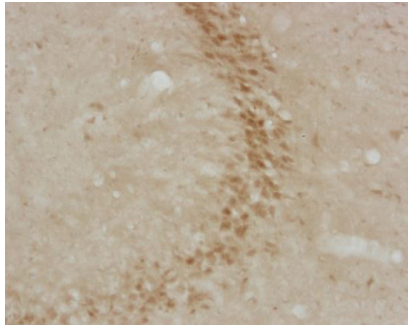

(g)

FIgure 2: (a) The photograph of ChAT immunoreactivity on hippocampus. (b, e) normal group, (c, f) control group, and (d, g) Squid-PS group ChAT expression on the hippocampus. Each column represents the mean value \pm SEM per group. Statistics: one-way ANOVA test, followed by LSD test. ${ }^{* *} P<0.01,{ }^{* * *} P<0.001$ as compared with the corresponding data of normal group. ${ }^{++} P<0.01$ as compared with the corresponding data of control group.

avoidance task along with signs of neurodegeneration, including decreased cholinergic neurons and neural glucose activity in the hippocampus. Repeated treatment with SquidPS attenuated the TMT-induced learning and memory deficits in the passive avoidance task, and it had a protective effect against the TMT-induced decrease in cholinergic positive neurons. Also, Squid-PS increased the glucose uptake approximately twofold in the frontal lobe and hippocampus.

Intoxication with trimethyltin (TMT) leads to profound behavioral and cognitive deficits in both humans [30] and experimental animals $[7,10]$. TMT is known to be widely used as plastic stabilizers, wood preservatives, anti-corrosion coatings, pesticides, and kill snails, as well as applied by chemical disinfector and sterilization. So it is clinically meaningful that humans as well as animals can be exposed to it in living environment. In rats, TMT induced the degeneration of the pyramidal neurons in the hippocampus and the cortical areas (pyriform cortex, entorhinal cortex, and subiculum) connected to the hippocampus, but there was also neuronal loss in the association areas $[6,11,12,14,15]$. Furthermore, behavioral studies have shown increased disruption of the memory, and learning deficits of TMT-intoxicated rats [20]. TMT intoxication impairs the acquisition of water maze performance [31]. The memory 


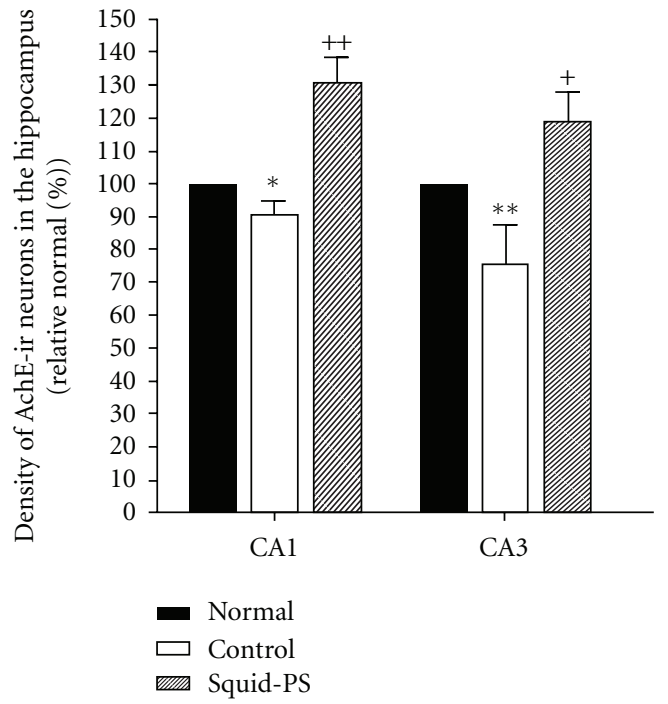

(a)

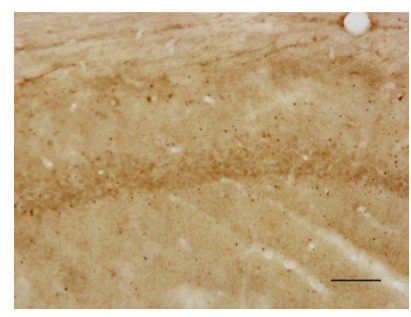

(b)

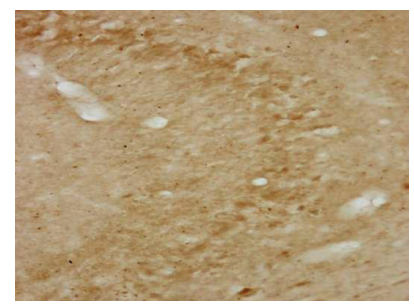

(e)

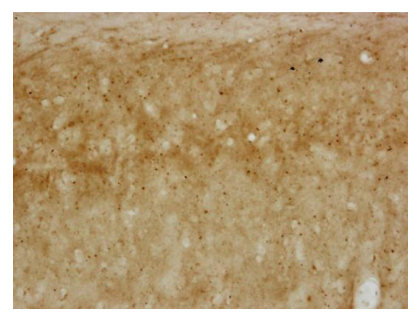

(c)

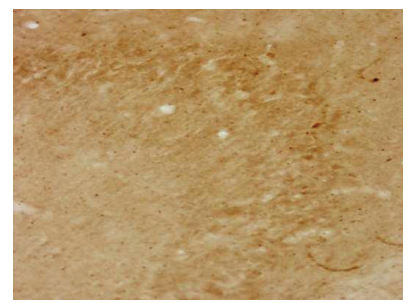

(f)

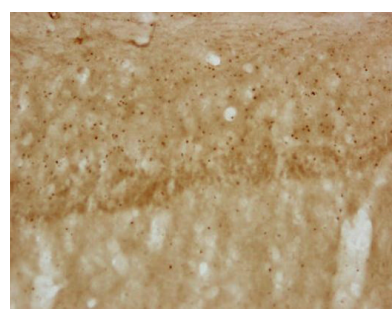

(d)

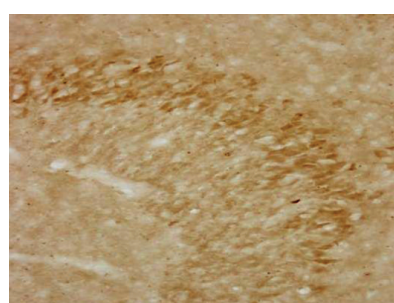

(g)

Figure 3: (a) The photograph of AchE immunoreactivity on hippocampus. (b, e) normal group, (c, f) control group, and (d, g) Squid-PS group AchE expression on the hippocampus. Each column represents the mean value \pm SEM per group. Statistics: one-way ANOVA test, followed by LSD test. ${ }^{*} P<0.05,{ }^{* *} P<0.01$ as compared with the corresponding data of normal group. ${ }^{+} P<0.05,{ }^{++} P<0.01$ as compared with the corresponding data of control group.

impairment produced by TMT in the current study is consistent with the previous reports of learning impairments [14, 31-33]. During the training trial in a step-through inhibitory avoidance task after shock, there were no differences among the groups. Treatment of Squid-PS seems to be more effective in reversing the memory impairment of late phase, rather than early phase, suggesting that it may facilitate process of memory consolidation. It has been previously reported that PS has profound curative effects on improving the memory and cognitive function of an Alzheimer's disease-like animal model [22, 34]. Zanotti et al. also showed that the treatment of PS in a scopolamine-induced animal model enhanced the learning and memory abilities of the rats [34]. But there was no study using Squid-PS in TMT intoxication model yet.
TABLE 1: It shows the results of voxelwise comparison between Squid-PS and control datasets. In SPM analysis, the cerebral glucose metabolism of Squid-PS datasets was increased significantly in the hippocampus and frontal lobe compared to control.

\begin{tabular}{lcc}
\hline Brain area & Coordinates $(x, y, z)$ & $Z$ value \\
\hline Frontal lobe & $(-2.4,2.7,8.1)$ & 2.14 \\
Hippocampus (Right) & $(7.5,2.1,2.4)$ & 2.85 \\
Hippocampus (Left) & $(-6.5,2.1,2.5)$ & 2.13 \\
\hline
\end{tabular}

The effects of Squid-PS on the central acetylcholine system were also examined by performing immunohistochemistry of the hippocampal neurons. The degeneration of 


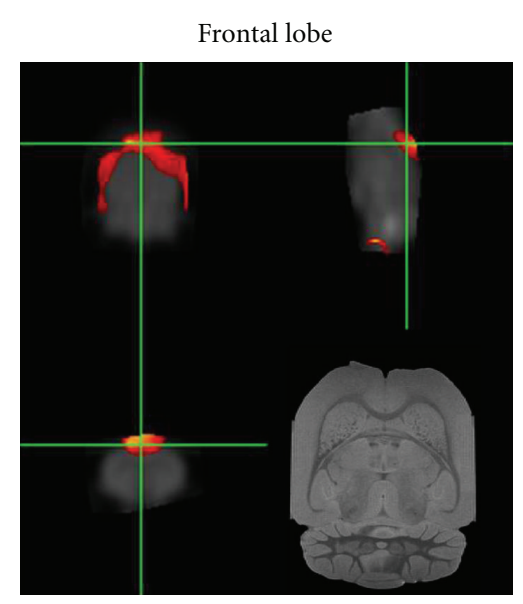

(a)

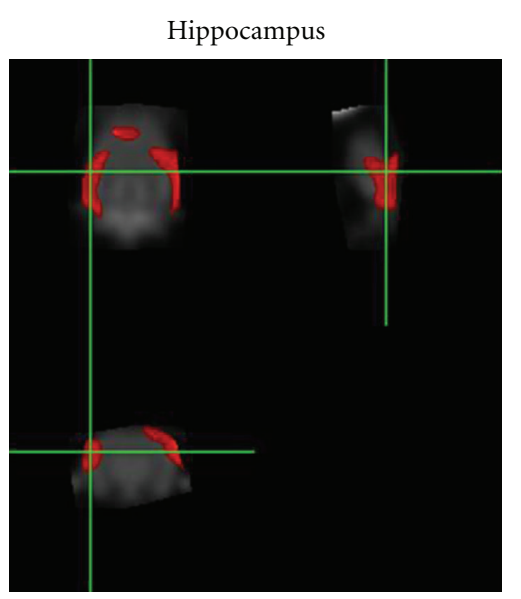

(b)

FIgURE 4: Brain regions where regional FDG uptakes in Squid-PS were significantly higher than in control (hippocampus and frontal lobe).

the cholinergic innervation from the basal forebrain to the hippocampal formation in the temporal lobe is thought to be one of the factors determining the progression of memory decay, both during normal aging and $\mathrm{AD}$ [35]. The best available marker for cholinergic neurons in the basal forebrain is ChAT activity. ChAT synthesizes the neurotransmitter acetylcholine in the basal forebrain, cortex, hippocampus, and amygdala. A significant reduction in ChAT activity in the postmortem brains of demented patients has been reported. In addition, there was a $20-50 \%$ decrease in ChAT activity in the hippocampus of the TMT-induced rats in this current study. However, the present results show that Squid-PS exerts beneficial effects on cholinergic neurotransmission in the brain by increasing the hippocampus ChAT activities. Also, acetylcholine esterase (AchE) is an enzyme which breaks down acetylcholine and is a well-known target and biomarker for memory dysfunction or dementia [20]. In AchE histochemistry, the Squid-PS group showed higher AchE reactivity in both hippocampal CA1 and CA3. These results are consistent with previous reports showing that the cholinergic neurons in the brain are involved in learning and memory in humans and animals $[36,37]$. In particular, the hippocampal cholinergic neurons are involved in the formation and maintenance of short-term working memory or retention and retrieval processes in long-term reference memory $[16,18,38-41]$. Based on a previous study, this result suggests that the Squid-PS treatment can promote the memory function.

The name "PET" comes from Positron Emission Tomography. It is a new scanning technique in medical research. Small animal PET experiments can be performed using a variety of dedicated small animal scanners (ATLAS, RatPET, microPET). It is a functional imaging modality at molecular level and provides valuable insights into biochemical, physiological, pathological or pharmacological process in vivo. Recent research efforts find its application in a wide area, ranging from basic insights into the normal physiology and disease processes to drug and radiotracer development and gene therapy. The present study showed that the PET analysis, the cerebral glucose metabolism of the Squid-PS datasets was significantly increased in the hippocampus and frontal lobe as compared to the control. An obvious limitation of our study is that the spatial resolution of the present micro-PET system is not high enough to permit more specific analysis of the activity changes within certain brain structures. Nevertheless, there have been several studies that have investigated the brain activity changes in small animals using micro-PET technology $[2,20]$. Thus, an important point of our study is that in spite of the limited spatial resolution of the micro-PET system, we were able to detect the TMT-induced focal brain changes.

In summary, treatment with Squid-PS attenuated the TMT-induced learning and memory deficits in the passive avoidance test, and Squid-PS treatment had a protective effect against a TMTinduced decrease of the cholinergic neurons and neural activation. Thus, Squid-PS is a good candidate of neuroprotective agent for treatment of Alzheimer's disease. Further studies that will examine the effects of Squid-PS activation on additional behavioral test will help to elucidate whether increasing the central cholinergic signaling may also improve other types of memory.

\section{Conflict of Interests}

The authors declare that there is no conflict of interests.

\section{Authors' Contribution}

Hyun-Jung Park and Seung Youn Lee are equally contributed to this work.

\section{Acknowledgments}

This research was supported by the Original Technology Research Program for Brain Science through the National Research Foundation of Korea (NRF) funded by the Ministry of Education, Science and Technology (2011-0030091) and this research was supported by a grant from the Korea 
Healthcare Technology R\&D Project, Ministry for Health, Welfare \& Family Affairs (Grant no. A091037), Republic of Korea.

\section{References}

[1] R. L. Cannon, D. B. Hoover, and M. L. Woodruff, "Trimethyltin increases choline acetyltransferase in rat hippocampus," Neurotoxicology and Teratology, vol. 13, no. 2, pp. 241244, 1991.

[2] A. O'Connell, B. Earley, and B. E. Leonard, "The neuroprotective effect of tacrine on trimethyltin induced memory and muscarinic receptor dysfunction in the rat," Neurochemistry International, vol. 25, no. 6, pp. 555-566, 1994.

[3] A. O'Connell, B. Earley, and B. E. Leonard, "Changes in muscarinic (M1 and M2 subtypes) and phencyclidine receptor density in the rat brain following trimethyltin intoxication," Neurochemistry International, vol. 25, no. 3, pp. 243-252, 1994.

[4] A. W. O'Connell, B. Earley, and B. E. Leonard, "The $\sigma$ ligand JO 1784 prevents trimethyltin-induced behavioural and $\sigma$ receptor dysfunction in the rat," Pharmacology and Toxicology, vol. 78, no. 5, pp. 296-302, 1996.

[5] C. Brabeck, F. Michetti, M. C. Geloso et al., "Expression of EMAP-II by activated monocytes/microglial cells in different regions of the rat hippocampus after trimethyltin-induced brain damage," Experimental Neurology, vol. 177, no. 1, pp. 341-346, 2002.

[6] C. D. Balaban, J. P. O'Callaghan, and M. L. Billingsley, "Trimethyltin-induced neuronal damage in the rat brain: comparative studies using silver degeneration stains, immunocytochemistry and immunoassay for neuronotypic and gliotypic proteins," Neuroscience, vol. 26, no. 1, pp. 337-361, 1988.

[7] R. S. Dyer, "Physiological methods for assessment of trimethyltin exposure," Neurobehavioral Toxicology and Teratology, vol. 4, no. 6, pp. 659-664, 1982.

[8] M. C. Geloso, V. Corvino, V. Cavallo et al., "Expression of astrocytic nestin in the rat hippocampus during trimethyltininduced neurodegeneration," Neuroscience Letters, vol. 357, no. 2, pp. 103-106, 2004.

[9] M. C. Geloso, A. Vercelli, V. Corvino et al., "Cyclooxygenase-2 and caspase 3 expression in trimethyltin-induced apoptosis in the mouse hippocampus," Experimental Neurology, vol. 175, no. 1, pp. 152-160, 2002.

[10] N. Ishida, M. Akaike, S. Tsutsumi et al., "Trimethyltin syndrome as a hippocampal degeneration model: temporal changes and neurochemical features of seizure susceptibility and learning impairment," Neuroscience, vol. 81, no. 4, pp. 1183-1191, 1997.

[11] D. Koczyk, M. Skup, M. Zaremba, and B. Oderfeld-Nowak, "Trimethyltin-induced plastic neuronal changes in rat hippocampus are accompanied by astrocytic trophic activity," Acta Neurobiologiae Experimentalis, vol. 56, no. 1, pp. 237-241, 1996.

[12] A. W. Brown, W. N. Aldridge, B. W. Street, and R. D. Verschoyle, "The behavioral and neuropathologic sequelae of intoxication by trimethyltin compounds in the rat," American Journal of Pathology, vol. 97, no. 1, pp. 59-81, 1979.

[13] L. W. Chang and R. S. Dyer, "Septotemporal gradients of trimethyltin-induced hippocampal lesions," Neurobehavioral Toxicology and Teratology, vol. 7, no. 1, pp. 43-49, 1985.

[14] R. L. Cannon, D. B. Hoover, R. H. Baisden, and M. L. Woodruff, "Effects of trimethyltin (TMT) on choline acetyltransferase activity in the rat hippocampus: influence of dose and time following exposure," Molecular and Chemical Neuropathology, vol. 23, no. 1, pp. 27-45, 1994.

[15] R. L. Cannon, D. B. Hoover, R. H. Baisden, and M. L. Woodruff, "The effect of time following exposure to trimethyltin (TMT) on cholinergic muscarinic receptor binding in rat hippocampus," Molecular and Chemical Neuropathology, vol. 23, no. 1, pp. 47-62, 1994.

[16] B. Earley, M. Burke, B. E. Leonard, C. J. Gouret, and J. L. Junien, "A comparison of the psychopharmacological profiles of phencyclidine, ketamine and (+) SKF 10,047 in the trimethyltin rat model," Neuropharmacology, vol. 29, no. 8, pp. 695-703, 1990.

[17] J. J. Hagan, J. H. M. Jansen, and C. L. E. Broekkamp, "Selective behavioural impairment after acute intoxiication with trimethyltin (TMT) in rats," NeuroToxicology, vol. 9, no. 1, pp. 53-74, 1988.

[18] R. B. Messing, V. Devauges, and S. J. Sara, "Limbic forebrain toxin trimethyltin reduces behavioral suppression by clonidine," Pharmacology Biochemistry and Behavior, vol. 42, no. 2, pp. 313-316, 1992.

[19] M. Segal, "Behavioral and physiological effects of trimethyltin in the rat hippocampus," NeuroToxicology, vol. 9, no. 3, pp. 481-489, 1988.

[20] H. S. Swartzwelder, R. S. Dyer, W. Holahan, and R. D. Myers, "Activity changes in rats following acute trimethyltin exposure," NeuroToxicology, vol. 2, no. 3, pp. 589-593, 1981.

[21] M. L. Woodruff, R. H. Baisden, and A. J. Nonneman, "Anatomical and behavioral sequelae of fetal brain transplants in rats with trimethyltin-induced neurodegeneration," NeuroToxicology, vol. 12, no. 3, pp. 427-444, 1991.

[22] T. J. Walsh, D. B. Miller, and R. S. Dyer, "Trimethyltin, a selective limbic system neurotoxicant, impairs radial-arm maze performance," Neurobehavioral Toxicology and Teratology, vol. 4, no. 2, pp. 177-183, 1982.

[23] M. L. Woodruff, R. H. Baisden, R. L. Cannon, J. Kalbfleisch, and J. N. Freeman, "Effects of trimethyltin on acquisition and reversal of a light-dark discrimination by rats," Physiology and Behavior, vol. 55, no. 6, pp. 1055-1061, 1994.

[24] S. Chen and K. W. Li, "Comparison of molecular species of various transphosphatidylated phosphatidylserine (PS) with bovine cortex PS by mass spectrometry," Chemistry and Physics of Lipids, vol. 152, no. 1, pp. 46-56, 2008.

[25] Z. Hossain, M. Konishi, M. Hosokawa, and K. Takahashi, "Effect of polyunsaturated fatty acid-enriched phosphatidylcholine and phosphatidylserine on butyrate-induced growth inhibition, differentiation and apoptosis in Caco-2 cells," Cell Biochemistry and Function, vol. 24, no. 2, pp. 159-165, 2006.

[26] J. G. Kay, M. Koivusalo, X. Ma, T. Wohland, and S. Grinstein, "Phosphatidylserine dynamics in cellular membranes," Molecular Biology of the Cell. In press.

[27] X. Bustamante-Marín, C. Quiroga, S. Lavandero, J. G. Reyes, and R. D. Moreno, "Apoptosis, necrosis and autophagy are influenced by metabolic energy sources in cultured rat spermatocytes," Apoptosis, vol. 17, no. 6, pp. 539-550, 2012.

[28] G. Paxinos, C. Watson, M. Pennisi, and A. Topple, "Bregma, lambda and the interaural midpoint in stereotaxic surgery with rats of different sex, strain and weight," Journal of Neuroscience Methods, vol. 13, no. 2, pp. 139-143, 1985.

[29] Q. Bao, D. Newport, M. Chen, D. B. Stout, and A. F. Chatziioannou, "Perfrmance evalution of the inveon dedicated PET preclinical tomograph based on the NEMA NU-4 standards," Journal of Nuclear Medicine, vol. 50, no. 3, pp. 401-408, 2009.

[30] K. Mizoguchi, M. Yuzurihara, A. Ishige, H. Sasaki, and T. Tabira, "Chronic stress impairs rotarod performance in rats: 
implications for depressive state," Pharmacology Biochemistry and Behavior, vol. 71, no. 1-2, pp. 79-84, 2002.

[31] B. Earley, M. Burke, and B. E. Leonard, "Behavioural, biochemical and histological effects of trimethyltin (TMT) induced brain damage in the rat," Neurochemistry International, vol. 21, no. 3, pp. 351-366, 1992.

[32] B. J. Fueger, J. Czernin, I. Hildebrandt et al., "Impact of animal handling on the results of 18F-FDG PET studies in mice," Journal of Nuclear Medicine, vol. 47, no. 6, pp. 999-1006, 2006.

[33] D. Koczyk, "How does trimethyltin affect the brain: facts and hypotheses," Acta Neurobiologiae Experimentalis, vol. 56, no. 2, pp. 587-596, 1996.

[34] A. Zanotti, L. Valzelli, and G. Toffano, "Chronic phosphatidylserine treatment improves spatial memory and passive avoidance in aged rats," Psychopharmacology, vol. 99, no. 3, pp. 316$321,1989$.

[35] B. Lee, B. J. Sur, J. J. Han et al., "Krill phosphatidylserine improves learning and memory in Morris water maze in aged rats," Progress in Neuro-Psychopharmacology and Biological Psychiatry, vol. 34, no. 6, pp. 1085-1093, 2010.

[36] C. N. Pope, B. T. Ho, and A. A. Wright, "Neurochemical and behavioral effects of N-ethyl-acetylcholine aziridinium chloride in mice," Pharmacology Biochemistry and Behavior, vol. 26, no. 2, pp. 365-371, 1987.

[37] H. Sun, Y. Hu, J.-M. Zhang, S.-Y. Li, and W. He, "Effects of one Chinese herbs on improving cognitive function and memory of Alzheimer's disease mouse models," China Journal of Chinese Materia Medica, vol. 28, no. 8, pp. 751-754, 2003.

[38] C. A. Cohen, R. B. Messing, and S. B. Sparber, "Selective learning impairment of delayed reinforcement autoshaped behavior caused by low doses of trimethyltin," Psychopharmacology, vol. 93, no. 3, pp. 301-307, 1987.

[39] I. Izquierdo, L. A. Izquierdo, D. M. Barros et al., "Differential involvement of cortical receptor mechanisms in working, short-term and long-term memory," Behavioural Pharmacology, vol. 9, no. 5-6, pp. 421-427, 1998.

[40] H. Kim, H. J. Park, H. S. Shim et al., "The effects of acupuncture (PC6) on chronic mild stress-induced memory loss," Neuroscience Letters, vol. 488, no. 3, pp. 225-228, 2011.

[41] K. Mizoguchi, M. Yuzurihara, A. Ishige, H. Sasaki, and T. Tabira, "Effect of chronic stress on cholinergic transmission in rat hippocampus," Brain Research, vol. 915, no. 1, pp. 108-111, 2001. 


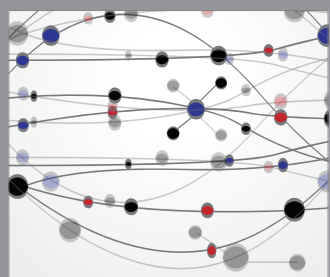

The Scientific World Journal
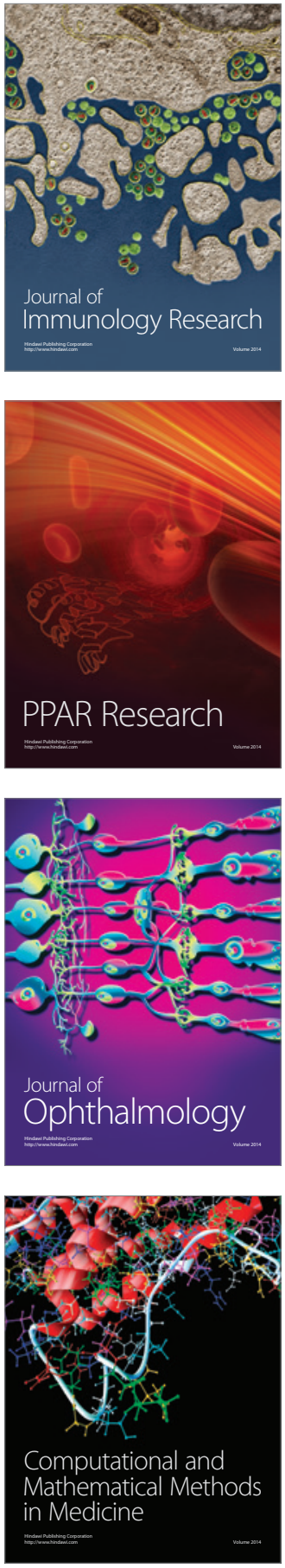

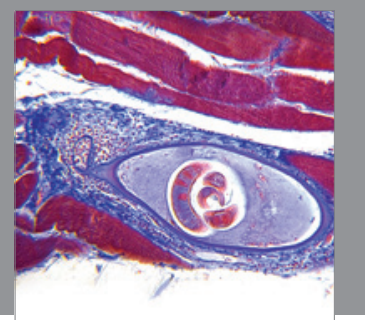

Gastroenterology

Research and Practice
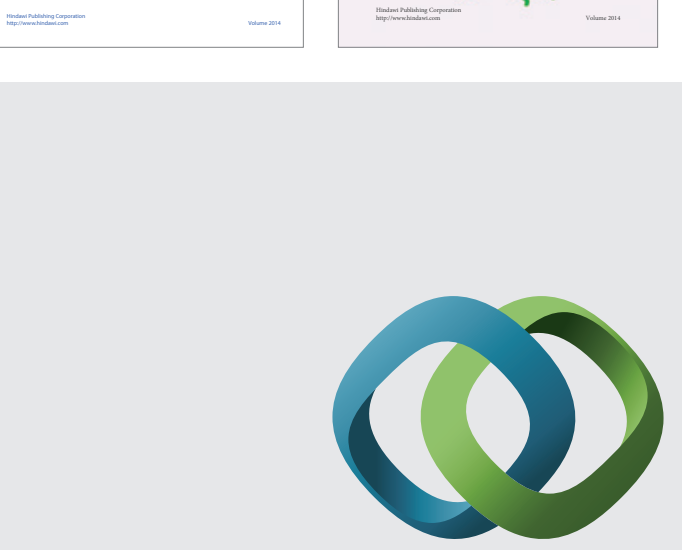

\section{Hindawi}

Submit your manuscripts at

http://www.hindawi.com
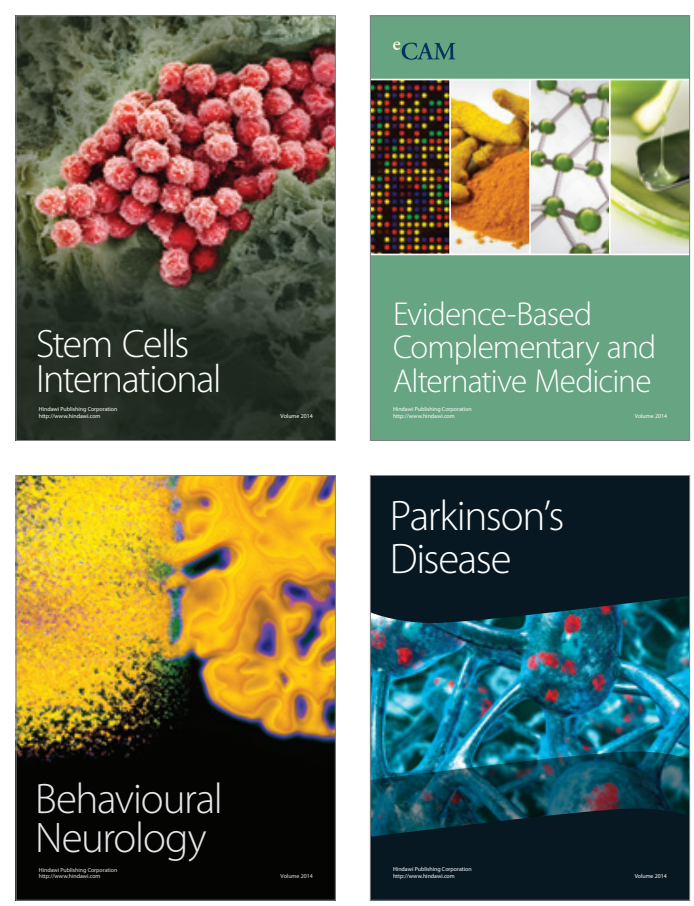

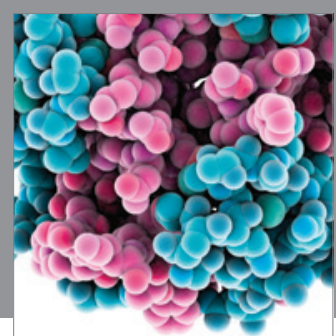

Journal of
Diabetes Research

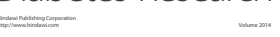

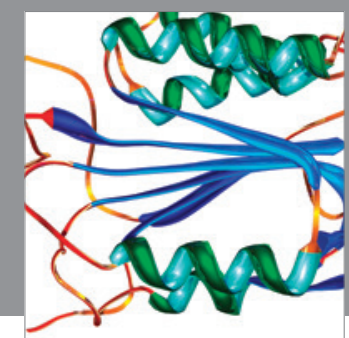

Disease Markers
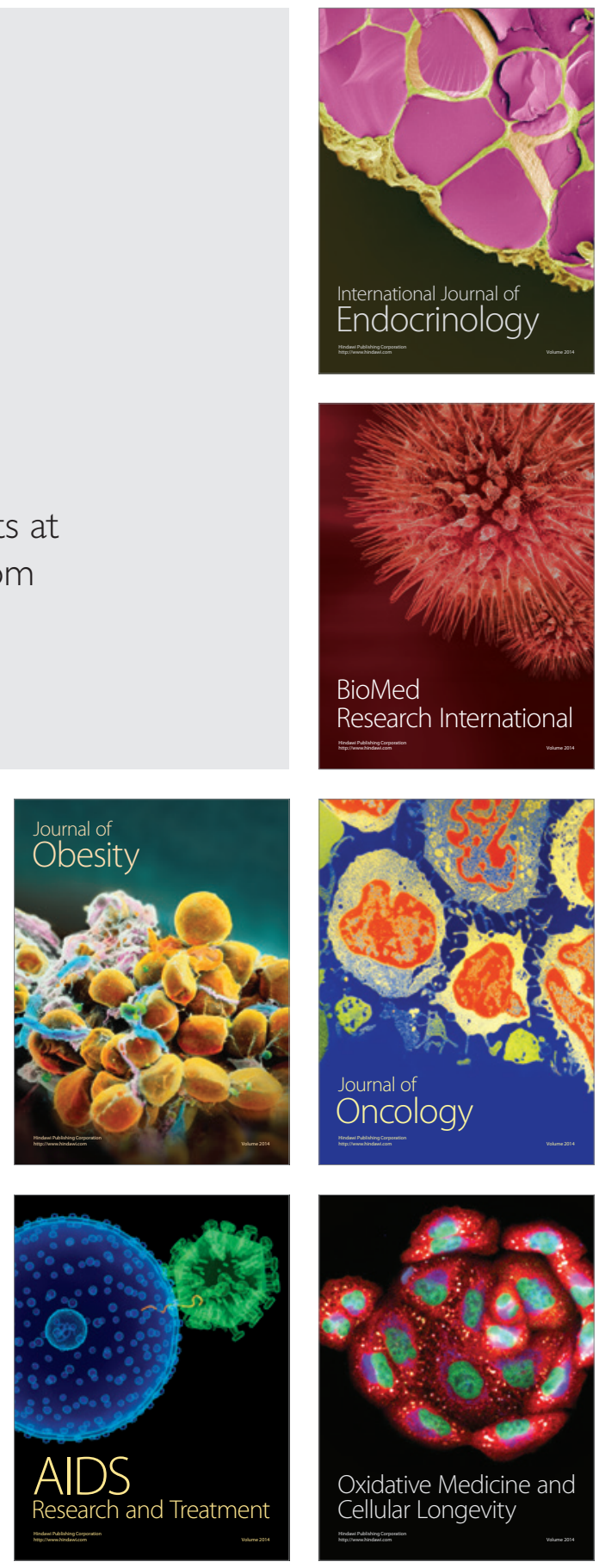